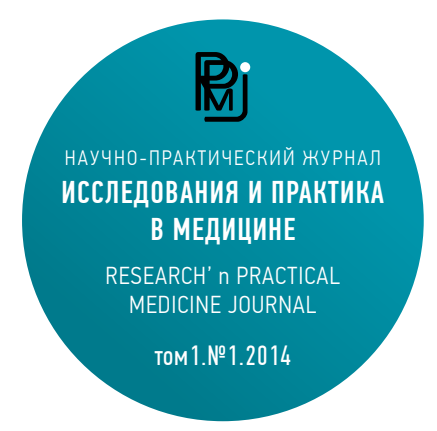

Ключевые слова:

рак молочной железы,

радикальная подкожная

мастэктомия, рецидивы

Keywords:

diabetes mellitus,

nonalcoholic fatty liver disease, cancer, obesity

\section{РЕЦИДИВЫ РАКА МОЛОЧНОЙ ЖЕЛЕЗЫ ПОСЛЕ ПОДКОЖНЫХ РАДИКАЛЬНЫХ МАСТЭКТОМИЙ С ОДНОМОМЕНТНОЙ РЕКОНСТРУКЦИЕЙ}

\author{
Зикиряходжаев А.Д., Рассказова Е.А.
}

МНИОИ им. П.А.Герцена — филиал ФГБУ «ФМИЦ им. П.А.Герцена» Минздрава России

(Москва, Российская Федерация)

125284, Российская Федерация, Москва, 2-ой Боткинский проезл, дом 3

\section{Резюме:}

Цели исследования. Проанализировать рецидивы после радикальных подкожных мастэктомий или кожесохраняющих мастэктомий.

Материалы и методы. В ФМИЦ им. П. А. Герцена пациенткам выполнена радикальная подкожная мастэктомия (95,5\%) или кожесохраняющая радикальная мастэктомия (4,5\%). При радикальной подкожной мастэктомии сохраняют кожу молочной железы, сосково-ареолярный комплекс (САК), субмаммарную складку, удаляют всю ткань железы вместе с подмышечными, подключичными и подлопаточными лимфатическими узлами. Кожесохраняющая мастэктомия отличается от подкожной мастэктомии удалением сосково-ареолярного комплекса. Распределение больных по стадии опухолевого процесса следующее: 0 (TisNO MO) - 33 (4,3\%), I - 180 (23,2\%), IIA - $283(36,6 \%)$, IIB - $123(15,9 \%)$, IIIA - 91 (11,7\%), IIIB - $1(0,1 \%)$, IIIC $41(5,3 \%)$ IY $-6(0,8 \%)$, у $16(2,1 \%)$ пациенток операция выполнена после рецидива РМЖ.

Основные результаты. В нашем исследовании рецидивы диагностированы у $37(4,8 \%)$ больных. Минимальный срок возникновения рецидивов 8 месяцев, максимальный 10 лет. Повторных местных рецидивов после проведенного лечения было 2.Местные рецидивы выявлены у 35 (4,5\%) больных. Регионарные рецидивы диагностированы у $3(0,4 \%)$ пациенток, в одном случае после местного рецидива. Регионарные рецидивы выявлены в $2(0,3 \%)$ случаях в подмышечной области и в $1(0,1 \%)$ случае в подключичной области. Вероятность возникновения регионарных рецидивов часто связывают с погрешностями в хирургической технике операции. При динамическом наблюдении выявлено $3(0,4 \%)$ случая развития рака Педжета в зоне сосково-ареолярного комплекса. Во всех 3 (0,4\%) случаях лечение было хирургическим и подразумевало иссечение сосково-ареолярного комплекса. У $1(0,1 \%)$ пациентки диагностирован РМЖ во второй молочной железе через 7 лет. В области сосково-ареолярного комплекса и в центральном квадранте рецидивы диагностированы в 6 из 35 локальных рецидивов, что составило 17,1\%. Среди пациенток с кожесохраняющей радикальной мастэктомией рецидива в центральном квадранте не выявлено. Распределение рецидивов в зависимости от стадии РМЖ: при I стадии - 5,5\%, при IIA - 3,5\%, при ІІБ - 5,7\%, при IIIA - 4,4\%, при IIIC - 7,3\%. В 2 случаях после первого рецидива из 16 диагностирован рецидив, что составило 12,5\%. При I стадии РМЖ диагностирован рецидив в 5,5\%, что вероятно связано с отсутствием лучевой терапии в послеоперационном периоде, т.к. стадия начальная и объем операции достаточен, при этом число рецидивов - 10 и ни в одном случае лучевую терапию не провели.

Выводы. За 5 лет наблюдений за больными раком молочной железы, после радикальных подкожных мастэктомий/кожесохраняющих радикальных мастэктомий с одномоментной реконструкцией, рецидивы развились у $34(4,4 \%)$ пациенток. Важным фактом является выявление местного рецидива после 10 лет наблюдения $(8,1 \%)$ после лечения, что подтверждает необходимость динамического наблюдения за пациентками в течение всей жизни. На частоту появления рецидивов влияет стадия РМЖ, молодой возраст пациенток, гистогенез и подтип опухоли. При сочетании неблаго- 
приятных прогностических факторов следует прибегать к реоперации на молочной железе. Радикальная подкожная мастэктомия или кожесохраняющая радикальная мастэктомия с одномоментной реконструкцией является адекватным объемом операции в онкологическом плане, а также эффективным методом реабилитации больных РМЖ. Развитие рецидива РМЖ после данных оперативных вмешательств не отличается от риска развития рецидива после радикальных мастэктомий. Несмотря на наличие повторной операции, сохранение реконструированной молочной железы после рецидива РМЖ было возможным в 65,7\% случаев в нашем исследовании.

\title{
RECURRENCE OF BREAST CANCER AFTER SUBCUTANEOUS MASTECTOMY WITH SIMULTANEOUS RECONSTRUCTION
}

\author{
Zikiryakhodzhaev A.D., Rasskazova E.A. \\ Moscow Oncology Institute of the Hertsen FMRC MH RF (Moscow, Russian Federation) \\ 3, 2 Botkinskiy proezd, 125284, Russian Federation, Moscow
}

\section{Abstract:}

Objective. To analyze the recurrence after radical subcutaneous mastectomy or konohana of mastectomy.

Materials and methods. In FMIC them.P. A. Herzen patients performed radical subcutaneous mastectomy $(95,5 \%)$ or konohana radical mastectomy (4.5 per cent). When radical subcutaneous mastectomy keep the skin of the breast, the nipple-areola complex (NAC), submammary fold, remove the entire gland tissue together with the axillary, subclavian and subscapularis lymph nodes. Konohana mastectomy differs from subcutaneous mastectomy with removal of the nipple-areola complex. Distribution of patients according to tumor stage process as follows: 0 (TisNOMO) - $33(4,3 \%)$, I - $180(23,2 \%)$, IIA - $283(36,6 \%)$, IIB - $123(15,9 \%)$, IIIA - $91(11,7 \%)$, III - $1(0,1 \%)$, IIIC - 41 $(5,3 \%)$ IY $-6(0,8 \%), 16(2,1 \%)$ patients the operation was performed after recurrence of breast cancer.

Results. In our study the recurrence was diagnosed in 37 $(4,8 \%)$ patients. A minimum of recurrences of 8 months, maximum 10 years. Repeated local recurrences after treatment was 2 . Local recurrence was diagnosed in $35(4,5 \%)$ patients. Regional recurrence was diagnosed in $3(0,4 \%)$ of patients, in one case after local recurrence. Regional recurrences detected in $2(0,3 \%)$ cases in the armpit and 1 $(0,1 \%)$ case in the infraclavicular region. The likelihood of regional recurrence is often associated with errors in surgical technique the operation. During follow-up revealed $3(0,4 \%)$ cases of cancer Paget's disease in the area of the nipple-areola complex. All $3(0,4 \%)$ of cases the treatment was surgical and meant excision of the nipple-areola complex. In $1(0,1 \%)$ patients diagnosed with breast cancer in the second breast after 7 years.In the area of the nipple-areola complex in the Central quadrant of the recurrence was diagnosed in 6 of the 35 local recurrence, which amounted to $17,1 \%$. Among patients with konohana radical mastectomy recurrence in the Central quadrant is not revealed. The distribution of recurrence depending on the stage of breast cancer: in stage I of 5,5\%, IIA - 3,5\%, IIB $-5,7 \%$, IIIA $-4,4 \%$ in $S$ of $7,3 \%$. In 2 cases after the first relapse of 16 was diagnosed with a relapse, which was $12.5 \%$. In stage I breast cancer was diagnosed with a relapse of $5,5 \%$, which is probably due to the absence of

radiation therapy in the postoperative period, since the initial stage and the operation volume is sufficient, the number of relapse - 10 and in any case, radiation therapy was not carried out.

Conclusion. Over the 5 years of observation for patients with breast cancer after radical subcutaneous mastectomy/konohana radical mastectomy with simultaneous reconstruction, recurrence developed in 34 cases (4.4\%) patients. The important fact is the detection of local recurrence after 10 years of follow-up $(8,1 \%)$ after treatment, which confirms the need for dynamic monitoring of patients throughout life. The frequency of relapses affected by stage of breast cancer, young age of patients, the histogenesis and subtype of the tumor. When combined adverse prognostic factors should be used for reoperation on the breast.

Radical subcutaneous mastectomy or konohana radical mastectomy with simultaneous reconstruction of an adequate volume of transactions in the cancer plan, and effective method of reabilitacii breast cancer patients. Relapse of breast cancer after these surgeries is not different from the risk of recurrence after radical mastectomy. Despite the presence of repeated operations, the preservation of the reconstructed breast cancer after recurrence of breast cancer was possible in $65,7 \%$ of cases in our study. 


\section{Введение}

Лечение рака молочной железы (РМЖ) подразумевает оперативное вмешательство, лучевую, системную, таргетную и гормональную терапию. В зависимости от стадии РМЖ, биологических особенностей опухоли, иммунного статуса пациентки зависит дальнейший период ремиссии болезни, а именно вероятность развития рецидива РМЖ. РМЖ хроническое системное заболевание $[1,2,3]$. Заболеваемость РМЖ имеет тенденцию к росту во всем мире.

Рецидивы РМЖ делят на местные/локальные (в молочной железе, в области послеоперационного рубца) и регионарные (поражение лимфатических узлов). Также, рецидив должен быть аналогичен гистологическому типу первичной опухоли $[4,5,6]$. Вероятность развития рецидива коррелирует со стадией болезни. Локальные рецидивы РМЖ наиболее часто, примерно в 75\% случаев, возникают впервые 5 лет после перенесенной операции [7]. Споры как влияет рецидив, на дальнейшее течение болезни продолжаются. Рецидив - это предсказатель риска генерализации РМЖ? И напротив рецидив не влияет на развитие болезни в последующем $[8,9]$.

Основным методом борьбы с уменьшением рецидива является лучевая терапия. Облучение всей молочной железы снижает риск местного рецидива на 2/3, а подведение дополнительного буста еще на $50 \%$ снижает риск развития рецидива $[4,9]$.

\section{Цель исследования}

Проанализировать рецидивы после радикальных подкожных мастэктомий или кожесохраняющих мастэктомий.

\section{Материалы и методы}

В ФМИЦ им. П. А. Герцена пациенткам выполнена радикальная подкожная мастэктомия или кожесохраняющая радикальная мастэктомия.

Для восстановления молочной железы применяли аутоткани или экспандеры/эндопротезы. Число больных 772 , у 2 (0,3\%) пациенток диагностирован первично-множественный синхронный рак молочных желез, таким образом, число операций 774.
Кожесохраняющая радикальная мастэктомия выполнена в (4,5\%) случаях, в 739 (95,5\%) радикальная подкожная мастэктомия. Единственное различие в данных операциях сохранение или удаление сосково-ареолярного комплекса. При этом кожу молочной железы, субмаммарную складку сохраняют. Объем лимфодиссекции зависит от поражения или отсутствия метастазов в лимфатических узлах.

Реконструкция утраченного объема молочной железы в 75\% комбинированная с использованием эндопротеза и в 25\% использованы аутоткани пациентки.

Медиана наблюдения за пациентками 16 лет (с 1997 по 2013 годы). Средний возраст пациенток $46 \pm 1,9$ лет (от 25 до 63 лет). Распределение больных по стадии опухолевого процесса следующее: 0 (TisNO MO) - 33 (4,3\%), I - 180 (23,2\%), IIA - $283(36,6 \%)$, IIB - $123(15,9 \%)$, IIIA - 91 (11,7\%), IIIB - 1 (0,1\%), IIIC $41(5,3 \%)$ IY $-6(0,8 \%)$, у $16(2,1 \%)$ пациенток операция выполнена после рецидива РМЖ (табл. 1).

IY стадия рака молочной железы была установлена из-за метастазов в кости (4 (0,5\%) пациентки) и в 2 (0,3\%) случаях - диагностированы метастазы в печень.

В 16 (2,1\%) случаях по поводу РМЖ в анамнезе была выполнена радикальная резекция молочной железы, в последующем диагностирован рецидив, для лечения которого выполнена операция подкожная мастэктомия с одномоментной реконструкцией.

В зависимости от стадии РМЖ по стандарту лечения выполняют лучевую терапию, а также курсы полихимиотерапии. Число курсов полихимиотерапии составило от 4 до 8. В случае гормонопозитивной опухоли назначают гормонотерапию. При экспрессии рецепторов Her2/neu 3+ назначают таргетную терапию по показаниям - герцептин, лапатиниб до 1 года приема [3].

Для диагностики рецидивов необходимо: клиническое обследование, ультразвуковое исследование и маммография. Наличие эндопротеза не препятствует выявлению местного рецидива.

Местный рецидив РМЖ: около 1/3 всех случаев рецидивов выявляют при обычном самообследовании, 1/3 при диагностической маммографии, 1/3 рециди-

Таблица 1

Распределение больных раком молочной железы в соответствиис системой TNM

\begin{tabular}{|c|c|c|c|}
\hline \multicolumn{2}{|l|}{ TNM } & \multirow{2}{*}{$\begin{array}{l}\text { Число больных (абс.) } \\
33\end{array}$} & \multirow{2}{*}{$\begin{array}{l}\text { Число больных (\%) } \\
4,3 \\
\end{array}$} \\
\hline & TisNOMO & & \\
\hline I CT & T1N0M0 & 180 & 23,2 \\
\hline IIA ст & T1-2No-1MO & 283 & 36,6 \\
\hline ІІБ ст & T2-3NO-1MO & 123 & 15,9 \\
\hline IIIA CT & TO-3N1-2MO & 91 & 11,7 \\
\hline ІІІБ ст & T4NO-2MO & 1 & 0,1 \\
\hline IIIC cT & T1-4N3M0 & 41 & 5,3 \\
\hline IY CT & T1-4N0-3M1 & 6 & 0,8 \\
\hline \multicolumn{2}{|c|}{ Рецидивы РМЖ } & 16 & 2,1 \\
\hline \multicolumn{2}{|l|}{ Bcero: } & 774 & 100,0 \\
\hline
\end{tabular}


вов - только при сочетании УЗИ и маммографии. Местный рецидив у $80 \%$ пациенток не сопровождается другими симптомами.

Для подтверждения рецидива РМЖ выполняют биопсию или цитологическое исследование пунктата узлового образования. А также при подтверждении рецидива проводится расширенное обследование, включая при необходимости магнитно-резонансную томографию и позитронно-эмиссионную ютомографию.

Традиционно под рецидивами РМЖ подразумевают возобновление опухолевого роста в молочной железе и в зонах расположения регионарных лимфатических узлов. Нередко указывают на характер роста рецидива: одиночный или множественный, узловой или диффузный (он же - лимфангитический, инфильтративный, панцирный).

\section{Результаты}

В нашем исследовании рецидивы диагностированы у 37 (4,8\%) больных. Минимальный срок возникновения рецидивов 8 месяцев, максимальный 10 лет. Повторных местных рецидивов после проведенного лечения было 2. Местные рецидивы выявлены у 35 (4,5\%) больных. Регионарные рецидивы диагностированы у $3(0,4 \%)$ пациенток, в одном случае после местного рецидива. Регионарные рецидивы выявлены в 2 (0,3\%) случаях в подмышечной области и в $1(0,1 \%)$ случае в подключичной области. Вероятность возникновения регионарных рецидивов часто связывают с погрешностями в хирургической технике операции.

При динамическом наблюдении выявлено 3 (0,4\%) случая развития рака Педжета в зоне сосково-ареолярного комплекса. Во всех $3(0,4 \%)$ случаях лечение было хирургическим и подразумевало иссечение сосково-ареолярного комплекса. У 1 (0,1\%) пациентки диагностирован РМЖ во второй молочной железе через 7 лет. В области сосково-ареолярного комплекса и в центральном квадранте рецидивы диагностированы в 6 из 35 локальных рецидивов, что составило 17,1\%. Среди пациенток с кожесохраняющей радикальной мастэктомией рецидива в центральном квадранте не выявлено.

По данным литературы известно, что лечение рецидива не отличается от лечения первичной опухоли. При необходимости используют арсенал, как местного, так и системного воздействия [8,3].

Лечение больных в 36 случаях было хирургическим, и только у одной пациентки в связи с одномоментным развитием локального рецидива и отдаленных метастазов было системное лечение (курсы полихимиотерапии).

В табл. 2 представлено время возникновения реци-

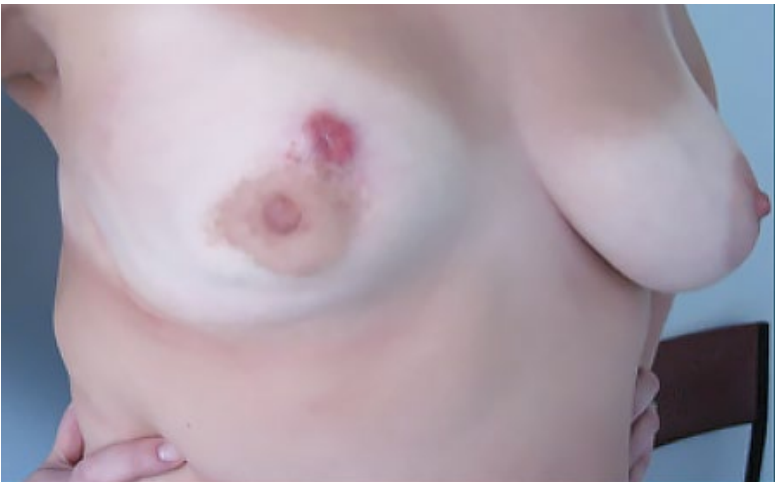

Рисунок 1. Местный рецидив рака правой молочной железы через 3 года после радикальной подкожной мастэктомии.

дивов от периода лечения до возникновения рецидива - подавляющее число рецидивов диагностировано до 5 лет - 91,9\%.

Изучение зависимости возникновения рецидива от гистогенеза опухоли выявило следующую закономерность: инвазивный протоковый рак II степени злокачественности в 20 (54,1\%) случаях, инвазивный протоковый рак III степени злокачественности - 8 (21,6\%), комбинированный рак - $7(18,9 \%)$, слизистый рак $2(5,4 \%)$.

В 14 (37,8\%) случаях неблагоприятными морфологическими признаками прогноза возникновения рецидива были раковые эмболы в сосудах вблизи опухолевого узла, рост инвазивного рака, c-r in situ вблизи опухолевого узла.

В $13(35,1 \%)$ случаях рецидивы возникли у женщин молодого возраста (до 40 лет), в 24 (64,9\%) после 40 лет. У женщин молодого возраста риск возникновения рецидива выше и составил 6,2\%, у женщин после 40 лет - 4,2\%.

Распределение рецидивов в зависимости от стадии РМЖ: при I стадии - 5,5\%, при IIA - 3,5\%, при ІІБ 5,7\%, при IIIA - 4,4\%, при IIIC - 7,3\%. В 2 случаях после первого рецидива из 16 диагностирован рецидив, что составило 12,5\%. При I стадии РМЖ диагностирован рецидив в 5,5\%, что вероятно связано с отсутствием лучевой терапии в послеоперационном периоде, т. к. стадия начальная и объем операции достаточен, при этом число рецидивов - 10 и ни в одном случае лучевую терапию не провели. Лечение рецидивов РМЖ было следующим: в 2,7\% случаев проведены курсы полихимиотерапии, так как рецидив в молочной железы был диагностирован одномоментно с отдаленными метастазами, в 97,3\% выполнены операции - от иссечения рецидива до удаления реконструированной молочной железы. В 3 случаях

Таблица 2

Время возникновения рецидивов РМЖ

\begin{tabular}{l|l}
\hline Время возникновения рецидивов & Число рецидивов \\
\hline До 3 лет & 24 \\
\hline $3-5$ лет & 10 \\
\hline $5-10$ лет & 3 \\
\hline Всего: & 37 \\
\hline
\end{tabular}


при иссечении рецидива дополнительно с целью абластики применили фотодинамическую терапию.

У 51,6\% пациенток проведена лучевая терапия после хирургического лечения рецидива, лекарственная терапия в 92,6\% случаев. Несмотря на наличие повторной операции сохранение реконструированной молочной железы после рецидива РМж было возможным в 65,7\% случаев, что несомненно повышает качество жизни пациенток даже после рецидива.

\section{Заключение}

За 5 лет наблюдений за больными раком молочной железы, после радикальных подкожных мастэктомий/кожесохраняющих радикальных мастэктомий с одномоментной реконструкцией, рецидивы развились у 34 $(4,4 \%)$ пациенток.

Важным фактом является выявление местного рецидива после 10 лет наблюдения (8,1\%) после лечения, что

\section{Список литературы}

1. Захарков Л.И. Подкожные радикальные мастэктомии с первичной реконструкцией при лечении больных раком молочной железы//Диссертация ... кандидата медицинских.наук: 14.00.14. - М: [б. н.], 2006 г.. - стр. 113.

2. Куклин И.А. Оптимизация хирургического лечения и реабилитации при опухолях молочной железы//Автореф. дис. ... д. М.Н.. - Иркутск: [б. н.], 2009 г..- стр. 35.

3. Kris M.G., Benowitz S.I., Adams S. Kris M.G., Benowitz S.I., Adams S., et al. Clinical cancer advances 2010: annual report on progress against cancer from the American Society of Clinical Oncology [Journal]//J Clin Oncol. - 2010. - Vol. 28. - pp. 53275347

4. Канаев С. В. Роль лучевой терапии в лечении рака молочной железы [Журнал]//Практическая онкология.- 2002 г..- 1: Т. 3. - стр. 45-51.

5. Криворотько П. В., Семиглазов В.В., Канаев С. В. и др. Факторы, влияющие на развитие местного рецидива после органосохраняющих операций на молочной железе//VIII Международная ежегодная конференция Проблемы диагностики и лечения рака молочной железы.- С-П: [б.н.], 2011 г.. - стр. 21-31.

6. Slavin S.A., Schnitt S.J., Duda R. B. et al. Skin-sparing mastectomy and immediate reconstruction: oncologic risk and aesthetic results in patients with early-stage breast cancer [Journal]//Plast Reconstr Surg. - 1998. - Vol. 102. - pp. 49-62.

7. Clarke M., Collins R., Darby S., et al. Early Breast Cancer Trialists' Collaborative Group (EBCTCG). Effects of radiotherapy and of differences in the extent of surgery for early breast cancer on local recurrence and 15-year survival: an overview of the randomisedtrials [Journal]//Lancet. - 2005. - Vol. 366. - pp. 2087-2106.

8. Молодикова Н.Р. Рецидивы рака молочной железы после органосохраняющего лечения//Диссертация ... кандидата медицинских наук. - 2010 г. - стр. 119.

9. Хмелевский Е.В. Современная лучевая терапия в лечении местнораспространенного и рецидивирующего рака молочной железы//Дисс. ... д. м.н. - 1997 г. - стр. 222.

Информация о соавторе:

1. Зикиряходжаев Азиз Дильшодович, доктор медицинских наук, руководитель отделения онкологии и реконструктивно-пластической хирургии молочной железы и кожи МНИОИ им. П.А.Герцена - филиал ФГБУ «ФМИЦ им. П.А.Герцена» Минздрава России подтверждает необходимость динамического наблюдения за пациентками в течение всей жизни.

На частоту появления рецидивов влияет стадия РМЖ, молодой возраст пациенток, гистогенез и подтип опухоли. При сочетании неблагоприятных прогностических факторов следует прибегать к реоперации на молочной железе.

Радикальная подкожная мастэктомия или кожесохраняющая радикальная мастэктомия с одномоментной реконструкцией является адекватным объемом операции в онкологическом плане, а также эффективным методом реабилитации больных РМЖ. Развитие рецидива РМЖ после данных оперативных вмешательств не отличается от риска развития рецидива после радикальных мастэктомий. Несмотря на наличие повторной операции, сохранение реконструированной молочной железы после рецидива РМЖ было возможным в 65,7\% случаев в нашем исследовании.

References

1. Zaharkov L.I. Podkozhnye radikal'nye mastjektomii s pervichnoj rekonstrukciej pri lechenii bol'nyh rakom molochnoj zhelezy//Dissertacija ... kandidata medicinskih.nauk: 14.00.14.M: [b. n.], 2006 g.. - str. 113.

2. Kuklin I. A. Optimizacija hirurgicheskogo lechenija i reabilitacii pri opuholjah molochnoj zhelezy//Avtoref. dis. ... d.m.n..Irkutsk: [b. n.], 2009 g.. - str. 35.

3. Kris M.G., Benowitz S.I., Adams S. Kris M.G., Benowitz S. I., Adams S., et al. Clinical cancer advances 2010: annual report on progress against cancer from the American Society of Clinical Oncology [Journal]//J Clin Oncol. - 2010. - Vol. 28. - pp. 53275347.

4. Kanaev S. V. Rol' luchevoj terapii $v$ lechenii raka molochnoj zhelezy [Zhurnal]//Prakticheskaja onkologija. - 2002 g.. - 1: T. 3. - str. 45-51.

5. Krivorot'ko P. V., Semiglazov V.V., Kanaev S. V. i dr. Faktory, vlijajushhie na razvitie mestnogo recidiva posle organosohranjajushhih operacij na molochnoj zheleze//VIII Mezhdunarodnaja ezhegodnaja konferencija Problemy diagnostiki i lechenija raka molochnoj zhelezy.-S-P: [b. n.], 2011 g.. - str. 21-31.

6. Slavin S.A., Schnitt S.J., Duda R.B. et al. Skin-sparing mastectomy and immediate reconstruction: oncologic risk and aesthetic results in patients with early-stage breast cancer [Journal]//Plast Reconstr Surg. - 1998. - Vol. 102. - pp. 49-62.

7. Clarke M., Collins R., Darby S., et al. Early Breast Cancer Trialists' Collaborative Group (EBCTCG). Effects of radiotherapy and of differences in the extent of surgery for early breast cancer on local recurrence and 15-year survival: an overview of the randomisedtrials [Journal]//Lancet. - 2005. - Vol. 366. - pp. 2087-2106.

8. Molodikova N.R. Recidivy raka molochnoj zhelezy posle organosohranjajushhego lechenija//Dissertacija ... kandidata medicinskih nauk. - 2010 g. - str. 119.

9. Hmelevskij E. V. Sovremennaja luchevaja terapija v lechenii mestnorasprostranennogo i recidivirujushhego raka molochnoj zhelezy//Diss. ... d. m.n. - 1997 g. - str. 222.

Information about co-author:

1. Zikiryakhodzhaev Aziz Dilshodovich, MD, director of the Department of Oncology and reconstructive plastic surgery of the breast and skin Moscow Oncology Institute of the Hertsen FMRC MH RF 